\title{
Permutations with short monotone subsequences
}

\author{
Dan Romik
}

Mathematical Sciences Research Institute, 17 Gauss Way, Berkeley, CA 94720-5070

\begin{abstract}
We consider permutations of $1,2, \ldots, n^{2}$ whose longest monotone subsequence is of length $n$ and are therefore extremal for the Erdős-Szekeres theorem. Such permutations correspond via the Robinson-Schensted correspondence to pairs of square $n \times n$ Young tableaux. We show that all the bumping sequences are constant and therefore these permutations have a simple description in terms of the pair of square tableaux. We deduce a limit shape result for the plot of values of the typical such permutation, which in particular implies that the first value taken by such a permutation is with high probability $(1+o(1)) n^{2} / 2$.
\end{abstract}

Keywords: Robinson-Schensted correspondence, Erdős-Szekeres theorem, limit shape

\section{Introduction}

In this note, we consider a class of permutations which have a certain extremality property with respect to the length of their monotone subsequences. The well-known Erdôs-Szekeres theorem states that a permutation $\pi=\left(\pi(1), \pi(2), \ldots, \pi\left(n^{2}\right)\right)$ of the numbers $1,2, \ldots, n^{2}$ must contain a monotone (either increasing or decreasing) subsequence $\pi\left(i_{1}\right), \pi\left(i_{2}\right), \ldots, \pi\left(i_{n}\right), \quad i_{1}<i_{2}<\ldots<i_{n}$. Our main object of study will be those permutations which do not have any longer monotone subsequences than those guaranteed to exist by this theorem.

Definition 1 A permutation $\pi \in \mathcal{S}_{n^{2}}$ is called an Extremal Erdôs-Szekeres (EES) permutation if $\pi$ does not have a monotone subsequence of length $n+1$. Denote by $E E S_{n}$ the EES permutations in $\mathcal{S}_{n^{2}}$.

The famous example showing sharpness of the Erdôs-Szekeres theorem is the permutation

$$
n, n-1, \ldots, 1,2 n, 2 n-1, \ldots, n+1,3 n, 3 n-1, \ldots, 2 n+1, \ldots, n^{2}, n^{2}-1, \ldots, n^{2}-n+1
$$

However, there are many more examples. Here is an EES permutation in $\mathcal{S}_{25}$ :

$$
13102015322232925172114781451611241918612
$$

It was observed by Knuth [1, Exer. 5.1.4.9] that the EES permutations in $E E S_{n}$ are in bijection with pairs of (standard) Young tableaux of square shape $(n, n, \ldots, n)$ via the Robinson-Schensted correspondence, and that, since the number of square Young tableaux can be computed using the hook formula of FrameRobinson-Thrall ([1, Th. 5.1.4.H]), this gives a formula for the number of EES permutations: 


$$
\left|E E S_{n}\right|=\left(\frac{\left(n^{2}\right) !}{1 \cdot 2^{2} \cdot 3^{3} \cdot \ldots \cdot n^{n} \cdot(n+1)^{n-1} \cdot(n+2)^{n-2} \cdot \ldots \cdot(2 n-1)^{1}}\right)^{2} .
$$

Our first result is a formula that describes the structure of an EES permutation in terms of a pair of square Young tableaux. This is a more precise description than that given by the Robinson-Schensted correspondence, which in general is quite difficult to analyze. First, introduce some useful notation. If a is a sequence of distinct numbers and $u$ is one of the numbers, denote

$$
\begin{aligned}
\operatorname{lis}(\mathbf{a}) & =\text { the maximal length of an increasing subsequence in } \mathbf{a}, \\
\operatorname{lds}(\mathbf{a}) & =\text { the maximal length of a decreasing subsequence in } \mathbf{a}, \\
\operatorname{lis}_{u}(\mathbf{a}) & =\text { the maximal length of an increasing subsequence in a containing } u, \\
\operatorname{lds}_{u}(\mathbf{a}) & =\text { the maximal length of a decreasing subsequence in a containing } u .
\end{aligned}
$$

Theorem 2 Let $\mathcal{T}_{n}$ be the set of square $n \times n$ standard Young tableaux. There is a bijection from $\mathcal{T}_{n} \times \mathcal{T}_{n}$ to $E E S_{n}$, defined as follows: to each pair of tableaux $P=\left(p_{i, j}\right)_{i, j=1}^{n}, \quad Q=\left(q_{i, j}\right)_{i, j=1}^{n}$ corresponds the permutation $\pi \in E E S_{n}$ given by

$$
\pi\left(q_{i, j}\right)=p_{n+1-i, j}, \quad(1 \leq i, j \leq n) .
$$

In the inverse direction, $P$ and $Q$ can be constructed from $\pi$ as follows:

$$
\begin{aligned}
& q_{i, j}=\text { the unique } 1 \leq k \leq n^{2} \text { such that } l d s_{\pi(k)}(\pi(1), \pi(2), \ldots, \pi(k))=i \text { and } \\
& l i s_{\pi(k)}(\pi(1), \pi(2), \ldots, \pi(k))=j . \\
& p_{i, j}=\text { the unique } 1 \leq k \leq n^{2} \text { such that } l d s_{\pi^{-1}(k)}\left(\pi^{-1}(1), \ldots, \pi^{-1}(k)\right)=i \text { and } \\
& \operatorname{lis}_{\pi^{-1}(k)}\left(\pi^{-1}(1), \ldots, \pi^{-1}(k)\right)=j .
\end{aligned}
$$

Next, we explore the properties of random EES permutations. For each $n$, let $\mathbb{P}_{n}$ be the uniform probability measure on $E E S_{n}$. If $\pi \in E E S_{n}$, define the plot of $\pi$ to be the set $A_{\pi}$ given by

$$
A_{\pi}=(i, \pi(i))_{1 \leq i \leq n^{2}}
$$

What does this set look like for a typical $\pi \in \mathcal{S}_{n}$ ? Figure 1(a) shows $A_{\pi}$ for a randomly chosen $\pi \in E E S_{100}$. For comparison, Figure 1(b) shows $A_{\pi}$ for a permutation $\pi$ chosen at random from all the permutations in $\mathcal{S}_{10000}$. Clearly the points in $A_{\pi}$ for a random EES permutation cluster inside a certain subset of the square $[1,10000] \times[1,10000]$. The phenomenon is explained by the following limit shape theorem, and is illustrated in Figure 2.

Theorem 3 Define the set

$$
\mathcal{Z}=\left\{(x, y) \in[-1,1] \times[-1,1]:\left(x^{2}-y^{2}\right)^{2}+2\left(x^{2}+y^{2}\right) \leq 3\right\} .
$$

Then: (i) For any open set $U$ containing $\mathcal{Z}$,

$$
\mathbb{P}_{n}\left[\pi \in E E S_{n}:\left(\frac{2}{n^{2}} A_{\pi}-(1,1)\right) \subset U\right] \underset{n \rightarrow \infty}{\longrightarrow} 1 .
$$




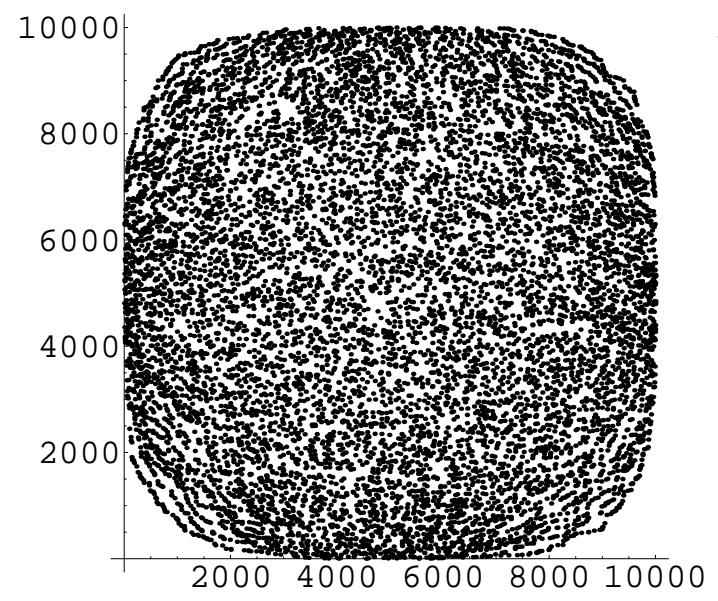

(a)

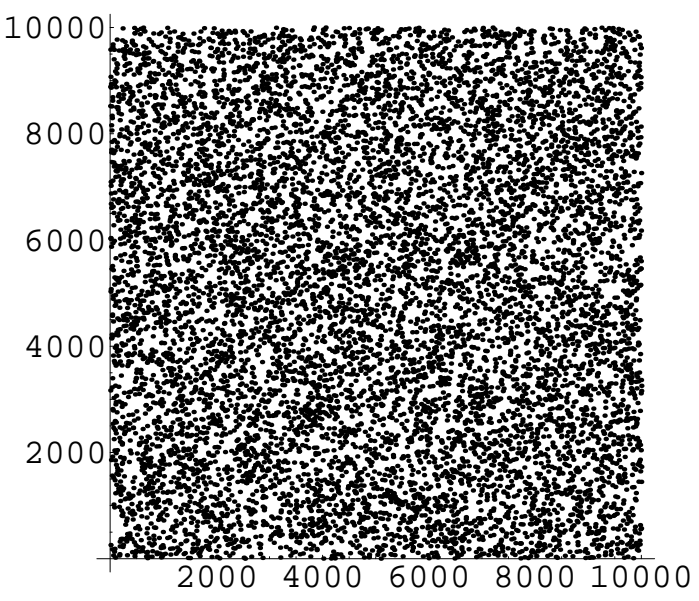

(b)

Fig. 1: A uniform random EES permutation and a uniform random permutation of $1,2, \ldots, 10000$.

(ii) For any open set $U \subset \mathcal{Z}$,

$$
\mathbb{P}_{n}\left[\pi \in E E S_{n}:\left(\frac{2}{n^{2}} A_{\pi}-(1,1)\right) \cap U \neq \emptyset\right] \underset{n \rightarrow \infty}{\longrightarrow} 1 .
$$

In particular, this implies the somewhat surprising fact that for large $n$, almost all EES permutations $\pi \in E E S_{n}$ satisfy $\pi(1) \approx n^{2} / 2$.

\section{Proof of Theorem 2}

In this section, we prove Theorem 2. Our proof uses the Robinson-Schensted correspondence. Although the bijection between EES permutations and pairs of square Young tableaux is a special case of the Robinson-Schensted correspondence, this special case is much simpler than the general case. For instance, the worst-case computational complexity of (1) is $O\left(n^{2}\right)$, and the worst-case complexity of (2) and (3) is $O\left(n^{2} \log n\right)$; compare this with the average-case complexity of $\theta\left(m^{3 / 2} \log m\right)$ of the RobinsonSchensted correspondence applied to a general permutation of $m$ elements (here $m=n^{2}$ ), see [3].

We assume that the reader is familiar with the definition and basic properties of the Robinson-Schensted correspondence; for background consult [1, section 5.1.4]. Recall that the Robinson-Schensted correspondence attaches to each permutation $\pi \in \mathcal{S}_{m}$ two standard Young tableaux $P$ and $Q$ whose shape is the same Young diagram $\lambda$ of size $m$. The length of the first row $\lambda_{1}$ of $\lambda$ is equal to lis $(\pi)$, and the length of the first column $\lambda_{1}^{\prime}$ of $\lambda$ is equal to lds $(\pi)$. In particular, if $\pi \in E E S_{n}$, then $\lambda$ is a Young diagram of size $n^{2}$ whose first row and column are both of length $n$; the only such diagram is the square diagram of shape $(n, n, \ldots, n)$, and this proves Knuth's observation mentioned in the introduction.

Our proof of (1) now relies on the following lemma. 


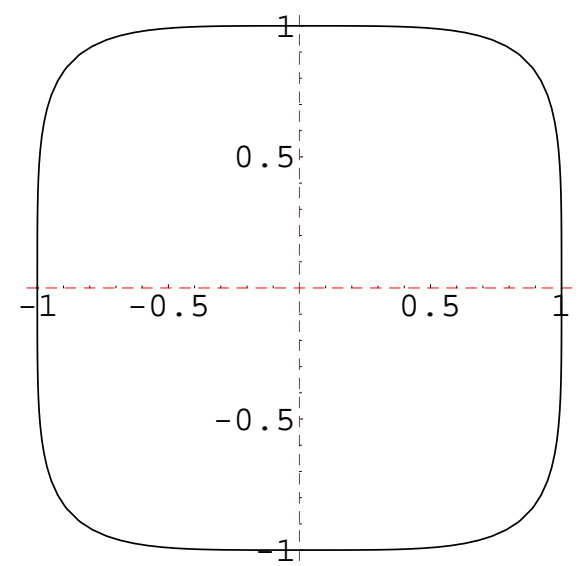

Fig. 2: The limiting shape of the plot of a random EES permutation. The boundary is the quartic curve $\left(x^{2}-y^{2}\right)^{2}+2\left(x^{2}+y^{2}\right)=3$.

Lemma 4 When the Robinson-Schensted correspondence is applied to an EES permutation $\pi \in E E S_{n}$ to compute the tableaux $P, Q$, all the bumping sequences are constant.

Proof We prove the obviously equivalent statement that in the application of the inverse RobinsonSchensted correspondence to two square $n \times n$ Young tableaux $P$ and $Q$, all the bumping sequences are constant. Recall that the inverse Robinson-Schensted correspondence consists of $n^{2}$ deletion operations, where at each step the corner element where the maximal entry in $Q$ is located is deleted from the shape of $P$ and $Q$, and $P$ is modified by bumping the entry of $P$ that was in the deleted corner up to the next higher row, then repeatedly bumping up an element from each row until reaching the top row.

The proof will be by induction on $k$, the number of deletion operations performed. For a given $k \geq 1$, let $\lambda$ be the shape of the tableaux $P$ and $Q$ after $k-1$ deletion operations (so $\lambda$ is the shape of the subtableau of the original $Q$ consisting of all entries $\left.\leq n^{2}-k+1\right)$. Denote by $P=\left(p_{i . j}\right)_{i, j=1}^{n}$ the entries of the original tableau $P$, and denote by $\hat{P}=\left(\hat{p}_{i, j}\right)_{i, j}$ the entries of the tableau $P$ after $k-1$ deletion operations. Assume that the $k$-th corner element to be deleted is at location $\left(i_{0}, j_{0}\right)$. A little reflection will convince the reader that the $k$-th bumping sequence will be constant if and only if for all $2 \leq i \leq i_{0}$ we will have that $\hat{p}_{i, j_{0}}<\hat{p}_{i-1, j_{0}+1}$ (where we take $\hat{p}_{i-1, j_{0}+1}=\infty$ if location $\left(i-1, j_{0}+1\right)$ lies outside $\lambda$ ).

By the induction hypothesis, all the bumping sequences before time $k$ were constant; another way to express this is via the equation

$$
\hat{p}_{i, j}=p_{i+n-\lambda^{\prime}(j), j}, \quad\left(1 \leq j \leq n, 1 \leq i \leq \lambda^{\prime}(j)\right),
$$

where $\lambda^{\prime}(j)$ is the length of the $j$-th column of $\lambda$, which simply says that the $j$-th row of $\hat{P}$ contains the $\lambda^{\prime}(j)$ bottom elements of the $j$-th row of $P$, in the same order. So we have

$$
\hat{p}_{i, j_{0}}=p_{i+n-\lambda^{\prime}\left(j_{0}\right), j_{0}}, \quad \hat{p}_{i-1, j_{0}+1}=p_{i-1+n-\lambda^{\prime}\left(j_{0}+1\right), j_{0}+1} .
$$

But $\left(i_{0}, j_{0}\right)$ is a corner element of $\lambda$, so $\lambda^{\prime}\left(j_{0}\right)=i_{0}>\lambda^{\prime}\left(j_{0}+1\right)$. This implies that $i+n-\lambda^{\prime}\left(j_{0}\right) \leq$ $i-1+n-\lambda^{\prime}\left(j_{0}+1\right)$, and therefore $\hat{p}_{i, j_{0}}<\hat{p}_{i-1, j_{0}+1}$, since $P$ is a Young tableau. 
Lemma 4 easily implies (1). At the $k$-th deletion step, if the corner cell being deleted is at location $\left(i_{0}, j_{0}\right)$ (so $\left.\lambda^{\prime}\left(j_{0}\right)=i_{0}\right)$, then $q_{i_{0}, j_{0}}=n^{2}-k+1$, and the element bumped out of the first row will be $\hat{p}_{1, j_{0}}=p_{n+1-\lambda^{\prime}\left(j_{0}\right), j_{0}}$. As a consequence we get $\pi\left(n^{2}-k+1\right)=\pi\left(q_{i_{0}, j_{0}}\right)=p_{n+1-i_{0}, j_{0}}$.

To conclude the proof of Theorem 2, we now prove (2) and (3). Clearly it is enough to prove (2), since replacing $\pi$ by $\pi^{-1}$ has the effect of switching $P$ and $Q$ in the output of the Robinson-Schensted correspondence. Note that $q_{i, j}=k$ if and only if $(i, j)$ was the corner cell that was added to the tableau $P$ at the $k$-th insertion step. Because of the properties of the Robinson-Schensted correspondence (specifically, [1, Th. 5.1.4.D(b)] and [1, Exer. 5.1.4.2]), this implies in particular that

$$
\operatorname{lds}_{\pi(k)}(\pi(1), \pi(2), \ldots, \pi(k)) \geq i, \quad \operatorname{lis}_{\pi(k)}(\pi(1), \pi(2), \ldots, \pi(k)) \geq j .
$$

Now, it is easy to see that

$$
\left\{\left(\operatorname{lds}_{\pi(k)}(\pi(1), \pi(2), \ldots, \pi(k)), \operatorname{lis}_{\pi(k)}(\pi(1), \pi(2), \ldots, \pi(k))\right): 1 \leq k \leq n^{2}\right\}
$$

is a set of distinct points in $\mathbb{Z}^{2}$ - this is the fact used in the well-known proof of the Erdôs-Szekeres theorem using the pigeon-hole principle (and this fact also justifies the use of the word "unique" in (2) and (3)). However, since $\pi$ is an EES, all these $n^{2}$ points lie in $[1, n] \times[1, n]$. So in fact the inequalities in (4) must be equalities, and (2) holds.

\section{Proof of Theorem 3}

We now prove Theorem 3. First, we recall the limit shape result for random square Young tableaux proved in [2]. For each $n \in \mathbb{N}$, let $\mu_{n}$ denote the uniform probability measure on $\mathcal{T}_{n}$, the set of $n \times n$ square Young tableaux. Pittel and Romik [2] proved that there is an (explicitly describable) function $L:[0,1] \times[0,1] \rightarrow[0,1]$ that describes the limiting surface of the typical square Young tableau (see Figure 3). More precisely:

Theorem 5 [2] For all $\epsilon>0$,

$$
\mu_{n}\left[T=\left(t_{i, j}\right)_{i, j=1}^{n} \in \mathcal{T}_{n}: \max _{1 \leq i, j \leq n}\left|\frac{1}{n^{2}} t_{i, j}-L(i / n, j / n)\right|>\epsilon\right] \underset{n \rightarrow \infty}{\longrightarrow} 0 .
$$

The only properties of the limit surface $L$ that we will need are that it is an increasing function of either coordinate, and that its values on the boundary of the square are given by

$$
L(t, 0)=L(0, t)=\frac{1-\sqrt{1-t^{2}}}{2}, \quad L(t, 1)=L(1, t)=\frac{1+\sqrt{2 t-t^{2}}}{2} .
$$

Let $\pi$ be a uniform random permutation in $E E S_{n}$. By Theorem 2, its plot can be described in terms of the tableaux $P$ and $Q$ (which are independent uniform random $n \times n$ square tableaux) by

$$
A_{\pi}=\left\{\left(q_{i, j}, p_{n+1-i, j}\right): 1 \leq i, j \leq n\right\} .
$$

By Theorem 5, each point $n^{-2}\left(q_{i, j}, p_{n+1-i, j}\right)$ is with high probability (as $\left.n \rightarrow \infty\right)$ uniformly close to the point $(L(u, v), L(1-u, v))$, where $u=i / n, v=j / n$. It follows that Theorem 3 is true with the limit shape set

$$
\mathcal{Z}^{\prime}=\{(2 L(u, v)-1,2 L(1-u, v)-1): 0 \leq u, v \leq 1\}
$$




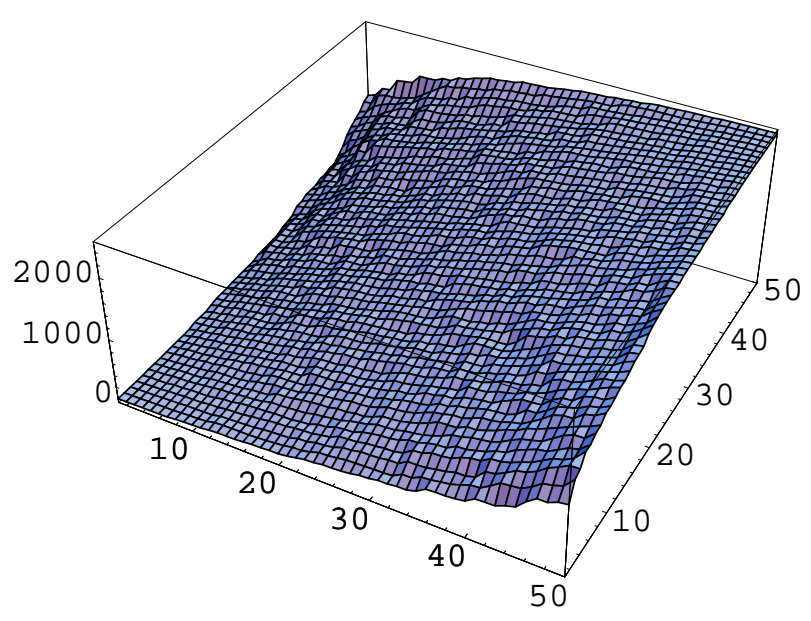

(a) 3D plot of a random tableau

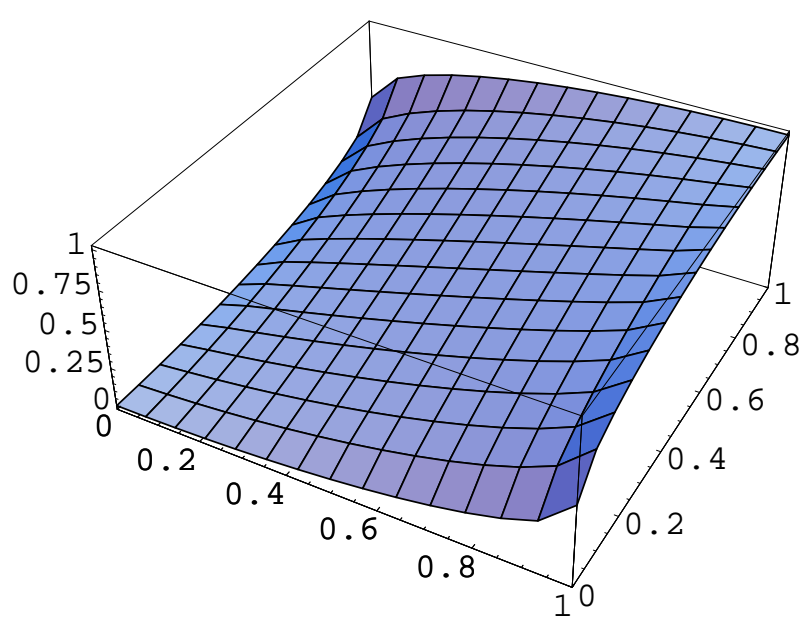

(b) The limit surface $L(x, y)$

Fig. 3: A random $50 \times 50$ square tableau and the limit surface

By (5), it follows that the mapping $(u, v) \rightarrow(2 L(u, v)-1,2 L(1-u, v)-1)$ maps the boundary of the square $[0,1] \times[0,1]$ into the four curves described parametrically by

$$
\begin{gathered}
\left(-\sqrt{1-t^{2}},-\sqrt{2 t-t^{2}}\right)_{0 \leq t \leq 1}, \quad\left(-\sqrt{1-t^{2}}, \sqrt{2 t-t^{2}}\right)_{0 \leq t \leq 1}, \\
\left(\sqrt{2 t-t^{2}}, \sqrt{1-t^{2}}\right)_{0 \leq t \leq 1}, \quad\left(\sqrt{2 t-t^{2}},-\sqrt{1-t^{2}}\right)_{0 \leq t \leq 1} .
\end{gathered}
$$

Setting $x= \pm \sqrt{2 t-t^{2}}, y= \pm \sqrt{1-t^{2}}$, it is easy to verify that

$$
\left(x^{2}-y^{2}\right)^{2}+2\left(x^{2}+y^{2}\right)=3,
$$

so these curves are the parametrizations of the boundary of the set $\mathcal{Z}$. It is also easy to check that the interior of the square is mapped to the interior of $\mathcal{Z}$, so $\mathcal{Z}^{\prime}=\mathcal{Z}$.

\section{References}

[1] D. E. Knuth, The Art of Computer Programming, vol. 3: Sorting and Searching, 2nd. ed. AddisonWesley, 1998.

[2] B. G. Pittel, D. Romik, Limit shapes for random square Young tableaux and plane partitions. Preprint, http://www.arxiv.org/abs/math.PR/0405190.

[3] D. Romik, The number of steps in the Robinson-Schensted algorithm. To appear in Funct. Anal. Appl. 39 (2005), no. 2. 